\title{
EMOTIONAL USER INTERFACES AND HUMANOID AVATARS IN INDUSTRIAL ENVIRONMENTS
}

\author{
Dirk Ziegeler, Detlef Zuehlke \\ Kaiserslautern University of Technology \\ Production Automation and Center for Human-Machine-Interaction \\ 67653 Kaiserslautern, Germany)
}

\begin{abstract}
With the increasing use of TFT monitors in machine controllers, the possibilities of interaction and visualization have changed considerably. On the same side modern production systems are getting more and more complex and a huge amount of information has to be visualized. To investigate the usefulness of virtual human-like characters - so called avatars - in industrial applications, a concept for visualization of specific information through different kinds of avatars within the same application will be presented. The specific character of a single avatar varies depending on the specific information to be presented. Copyright C 2005 IFAC
\end{abstract}

Keywords: Human factors, human reliability, interaction mechanisms, human-machineinterface, process control, feedback channel

\section{INTRODUCTION}

Today, most machines are equipped with a clear and simple mostly text-and-button user interface. Soon these will be replaced by the next generation of state of the art technology in user interfaces. Driven by the PC technology several devices like color TFT monitors or touch screens have become so powerful and cheap that they are more and more moving in machine or process controllers. Nowadays, the operator has to deal with the increased requirements of complex systems and has to get used to the more and more complex interaction with them. Naturally, these users are expecting a better assistance in terms of an optimized human-machine-interface. New display technologies, like touch- or semi transparent screens, allow new approaches for human-machineinteraction visual and haptical wise. Improved approaches in this field of research are developed by Peace (2003), Cao (2003) and Hachet (2002). A combination of new display technologies and artificial intelligence (Krzysztof, 2001), will enable the adaptation of the navigation/interface of a complex system based on the users behavior while using it.

Up till now emotions never played a role in humanmachine-systems. And this made sense because the old technologies were surely not able to offer affective interaction principles. But with today's sophisticated equipment using high-resolution color screens, artificial voice generators etc. we can no longer neglect emotions in human-machine-systems. Picard (2000) emphasized the upcoming significance of affective computing in terms of rethinking the human-machine-interaction. Using emotions as an interaction process with machines required a constitutive modification and creation of new strategies.

To increase the human factor in interacting with machines, several emotional acting avatars on all sorts of platforms where developed and refined till today (Cassell, 2000a), (Marsella, 2001), (Wahlster, 2001). Johnson (2000) accentuated the usefulness of a human like interface. He defined the condition and 
variables for a human-machine-interaction seen as a human-human-interaction.

Well known realizations of emotional avatars are found at user interfaces for office use (Cassell, 2000), (Wahlster, 2001). In the field of production or process applications the value of avatars was not tested yet. For future systems, the influence of human emotional behaviors (Picard, 2000) will be an outstanding requirement. Thus, avatars may play a key role for future emotional user interfaces.

\section{THE CHALLENGE OF AVATARS}

\subsection{Focus}

Today's systems are more and more highly automated and reliable. The operator tasks in such (badly designed) systems are reduced to survey the processes and stay in stand-by for unforeseen problems. If such problems occur the operator has to analyze the situation on the knowledge-based interaction level, which normally takes valuable time. Often, this leads to stress which then leads to a further reduction of the mental performance. When other people or friends are around, they evaluate the circumstances from a more distant point of view and can help with gestures or acclamations. In our concept avatars are used as virtual representatives for colleagues or people near by. They should act like a guide and support the operator in getting the right focus on what happens and how to return to a normal situation.

This new concept, using avatars on a machine interface, is clearly focused on industrial applications, including the standards and safety regulations within it. To investigate this new strategy, we created in the following a concept for visualization of information through different kinds of avatars. The specific character of the single avatar is linked to information classes like general information or alarm messages. We used obviously separated characters to present the different information levels as clear as possible. In the concept, the different avatars are seen as a supportive way to provide and communicate additional information to the user.

\subsection{AVATARS IN INDUSTRIAL ENVIRONMENTS}

With the use of color TFT monitors, the possibilities of interaction and visualization have changed considerably. On the same side manufacturing and process systems are getting more and more complex and a huge amount of information must be visualized on the UI. In daily business most complex user interfaces are working fine. Here, an avatar could bring "Joy-of-use" into the communication and motivate the user with friendly support and correct answers to create a positive work atmosphere.

Using emotions within this communication is a logical consequence. But emotions have two sides: the emotions of the user which have to be recognized, and the virtual emotions of the avatar which must be "engineered". Unfortunately, there is not much research yet to be found with regard to both requirements recognizing and engineering emotions in an engineering sense. The virtual representation of a human-like character showing the broad range of human emotions is still very hard to achieve. So, we decided to reduce this problem by using animated comic characters which show only few characteristical emotional expressions. We decided to realize a couple of multimodal affirmative signals, e.g. a kind of facial expression and nod of the head.

One of the main differences between an office and an industrial environment is the real-time requirement. Writing a letter normally is not a time-critical procedure, but an alert message from a safety-critical process will often require an action in seconds. Therefore, the adaptation of the interaction frequency and the occurrence of the avatars must be in accordance with the urgency of the upcoming actions. During an alarm message, the avatar communicates with explicit gestures and hand signals, clear and calm comments without any additional movements of the body. Everything is as short and clear as necessary - like in a real urgent situation. On the other hand, when the user needs some general information during normal process operation, the avatar is more a kind of a polite and courteous person, because the whole situation is straight forward and the user has time for entertainment, too.

\section{REQUISITIONS FOR AVATARS}

The trustworthiness of a character also plays an important role in a natural communication. Therefore, it is necessary to change the level of authority with the presented characters in a virtual representation as well. To accentuate these levels the outer appearance, voice characteristics, gesture and general movement will be different at each character. If the task of such an embodied agent is not consistent with his appearance, it is confirmed that it can be judged more negatively than an avatar which is coherent in its communication and his physically obtained impression. Attractive humans are awarded to more social authority, higher intelligence and a better psychological influence than less attractive. Agents, who must implement requests and rules or represent system experts, should possess accordingly ripe faces. Mature faces - with a stamped characteristic face, such as folds or salient outlines are classified as authoritative and technically competent. At the same time a female face is perceived more friendly and more helpfully than a male one.

Emotion-loaded faces have likewise an influence on the interpretation of a character. Easily rising corners of the mouth, like a natural smile, generally cause positive reactions with an association of smaller authority and dominance, than neutral or serious 
faces. The same influence applies to questioning faces with arise brows or sad facial expressions with low lying brows, thin lips or sloping corners of the mouth.

Heylen (2002) accentuated the fact that the manner of perception of a typical human-machine-interaction is changing to a situation of human-humancommunication at the side of the user as soon as an embodied avatar can be seen on the user interface. Johnson (2000) contributes that this perception establishes a higher motivation and readiness to work with the system due to the more true-to-life representation of the avatar. Johnson also mentioned that the simple appearance of an embodied avatar can create a pleasant working environment between the user and his machine.

But, how realistic or perfect do they have to be? To point it out, the acceptance of a specified character will not necessarily go along with the most natural visualization (Heylen, 2000). Naturalism and authenticity of anthropomorphic characters contradict themselves since a high percentage of perfection is required for a realistic representation. The acceptance and trustworthiness of the avatars will grow with a more naturalistic outside.

Empirical investigations (Aughenbaugh, 1999) have shown that users tend to change their typical behavior opposite of a machine to their typical behavior by a human, when a cartoon-like agent is shown on a panel. We combined these experiences into new ideas to create simpler but trustworthy avatars in an industrial application, which is described below.

Besides gestures, voice is also part of the natural communication process. Like the appearance, the voice also has to be adapted to the specific character. A warm and friendly tone or timbre is recommended for normal information and advisories. Avatars, which symbolize important system messages or execute rules, are interacting with a steady voice which is raised according to tendency. But, because of the relative high noise level in an industrial environment, the usage of voice communication will be limited.

Obviously, researchers have to consider several factors for the design of different characters. Like in everyday life, people are always looking for trustworthy personalities in case of an information demand. And they normally link specific characters to specific situations. Reflect this example: After a car accident, we first expect a policeman to appear and to clarify the situation. We may expect a nurse to help the injured and later a mechanic to repair the car. Subconsciously, the policeman is male, the nurse female and -for sure- the mechanic male again.

\section{SOLUTION}

Having the described experiences and environmental and process conditions in mind, this concept contains a complete family of characters to symbolize each category as best as possible. To visualize the different information classes, fig. 1 - 4 demonstrates some examples for potential characters. Typical stereotypes were used for the visualization of these characters, to achieve a high level of recognition by the user. Furthermore, each information class can be visualized by similar characters like a mechanic, a doctor or a teacher.

\section{General Information}

In the category of general information the user is in a straight forward working situation. He can focus the user interface with all his senses and has enough time to recognize each detail. The presented avatar should fit into this situation and motivate/invite the user to a more agile interaction with the interface, respectively the machine. For this communicative part of interaction, the avatar (fig. 1) needs a high potential on social authority and a motivating appearance.

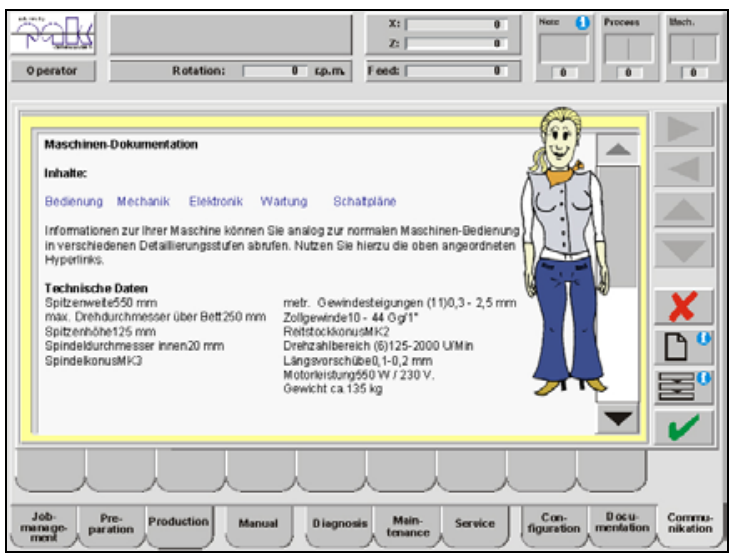

Fig. 1. Avatar for general information

\section{Maintenance information}

To transfer maintenance information, professional knowledge is the main requirement for the used avatar. Here, a young, male character dressed as a mechanic with a friendly charisma is preferred.

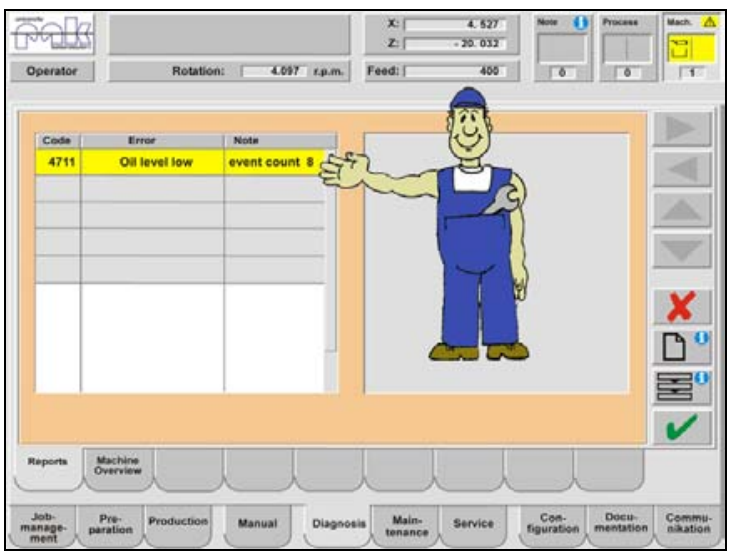

Fig. 2. Avatar for general information 


\section{Warning messages}

For a higher attentiveness, the character needs a higher authority and should have a mature appearance. He also has to implement requests and rules. To communicate these requirements at the first sight, the avatar has a stringent facial expression and wears a formal dress, like a uniform. This character visualizes all information in the warning message class and also when a severe misoperation is recognized.

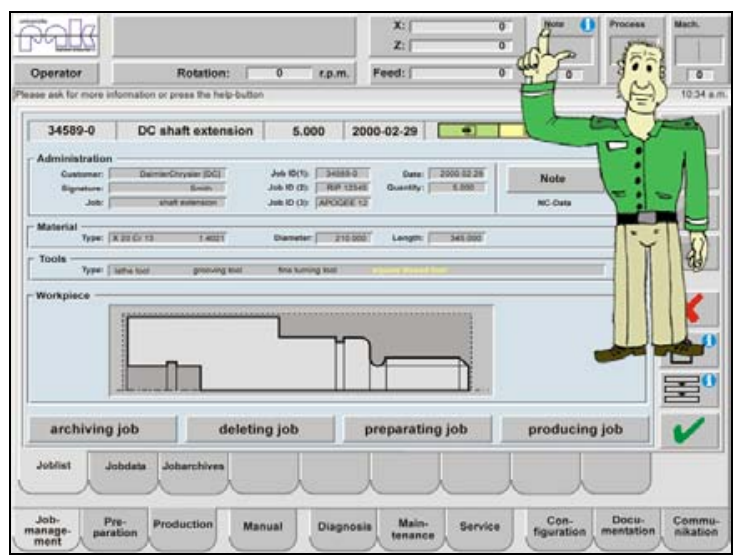

Fig. 3. Avatar for general information

\section{Alarm messages}

The highest level of messages are alarm messages, which require immediate actions. The avatar should symbolize this emergency situation. Here we need a very serious character which presents authority, technical skills and trustworthiness. We decided to use a fire fighter as an appropriate symbol.

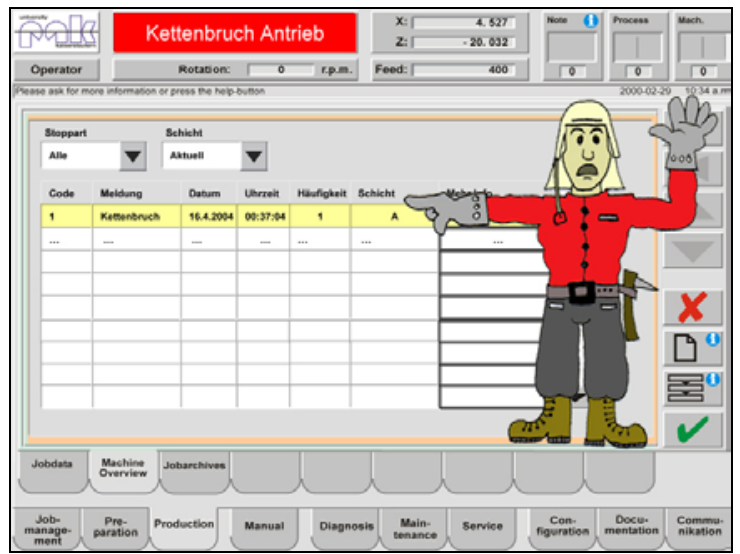

Fig. 4. Avatar presenting an alarm message

Further distinctions are achieved by different gestures in combination with vocal audio output. Additionally to verbal feedbacks the avatar can use a kind of head nodding and/or "thumb-up" signal as indication for an acknowledgment of correct actions or understanding.

For incorrect decisions or lacks of understanding, the avatar will show a confused facial expression in a rudimentary form. Gestures like a wince of the body strengthen the intensity of the feedback given in such a way.

\section{ACTION UNITS}

To describe the complex coherence between gesture, facial expressions and movements in detail, we developed for each chapter of information separate action units. Each unit describes an action with gestures, like a waving hand, body movements and facial expressions to get the attention of the user.

In order to clarify the different characteristics of the same message in different information classes, the following example is used:

An "error" message from a fire fighter (alarm information) will be much more forceful and resolute as from a young woman (general information). Because of the lower level of urgency, she can use a more polite form of linguistic and physical expression. These units are based on previous research of Ekman (1972) and Krämer \& Bente (2003) and adapted to the production environment. For example, we demonstrate the action unit "error" within the different information classes:

\begin{tabular}{|ll|}
\hline Action Unit ,error“ [General]: & Appease the user \\
\hline Face: & Time lapse: \\
surprised facial expression & at start for $1 \mathrm{sec}$. \\
friendly, but insistent & during interaction \\
be all smiles & to the end \\
\hline $\begin{array}{ll}\text { Body: } \\
\text { rightwards the head for } 10^{\circ}\end{array}$ & to the end for $3 \mathrm{sec}$. \\
\hline
\end{tabular}

\begin{tabular}{|ll|}
\hline $\begin{array}{l}\text { Action Unit ,error“ } \\
\text { user }\end{array}$ & $\begin{array}{l}\text { Time lapse: } \\
\text { at start for } 1 \mathrm{sec} .\end{array}$ \\
\hline $\begin{array}{l}\text { Face: } \\
\text { fise the brows }\end{array}$ & $\begin{array}{l}\text { all the time of the } \\
\text { interaction }\end{array}$ \\
smile & to the end \\
\hline $\begin{array}{l}\text { Body: } \\
\text { rightwards the head for } 10^{\circ}\end{array}$ & $\begin{array}{l}\text { Time lapse: } \\
\text { at the end for } 3 \mathrm{sec} .\end{array}$ \\
\hline
\end{tabular}

\begin{tabular}{|ll|}
\hline Action Unit ,error“ [Warning]: Appease the user \\
\hline Face: & Time lapse: \\
knit one's brows & at start \\
short smile & $\begin{array}{l}\text { at start and end of } \\
\text { interaction }\end{array}$ \\
neutral face & to the end \\
\hline Body: & Time lapse: \\
neutral & all the time \\
\hline
\end{tabular}




\begin{tabular}{|c|c|}
\hline \multicolumn{2}{|c|}{ Action Unit ,error" [Alarm]: Appease the user } \\
\hline Face: & Time lapse: \\
\hline rise the brows & at start \\
\hline serious expression & during interaction \\
\hline lift the corner of the mouth & to the end \\
\hline Body: & Time lapse: \\
\hline light shaking with the head & before start for $1 \mathrm{sec}$. \\
\hline neutral posture & all the time \\
\hline
\end{tabular}

Independent from the visual outline of the chosen character, developers can use these units as a guideline for must have features of their avatar.

\section{CHALLENGE \& PERSPECTIVES}

The described avatar-based interaction system was designed and installed over the last months. First labtests could be conducted, but many questions are still open to answer. We identified several challenges for further research in affective computing in the production environment with avatars.

- How will avatars improve the attentiveness of the user?

- How reliable will be the recognition of specific situations depending on the choice of specific characters and their emotional expression forms?

- Can we simplify process operation by using multimodal and natural communication?

- Will avatars contribute to a higher degree of safety in industrial applications?

- Which influences will culture have on the articulation and recognition of emotions?

- Is the individualization of interactive teaching programs and applications with anthropomorphic characters desirable?

- How is the long-term acceptance of such avatars by the users?

- Which efficiency is necessary to improve the job quality by admission of social aspects and adjustment of the communicative system?

\section{CONCLUSION}

Triggered by many new technologies, we will surely notice the use of avatars in future everyday applications like ticket vending machines or ATM's. Therefore, the question of using such technologies in industrial applications will also come up soon. The research work described in this paper should be seen as a necessary base for experimental work. In the next steps, user acceptance and attention control must be evaluated. Further on, the difference and efficiency between standard user interfaces and avatar-based UI's must be tested. This research is very interdisciplinary and on the long term culture dependant. Therefore, it will still need a lot of effort and time to move into industrial application.

\section{REFERENCES}

Aughenbaugh, J. (1999). Affective Issues in the Search of Artificial Intelligence. Princeton University, USA.

Cao, X., Balakrishnan, R. (2003). VisionWand: interactive techniques for large displays using a passive wand tracked 3D. In: Proceedings of the 16th annual ACM symposium on User interface software and technolog. Page 173-182. ACM Press, New York.

Cassell, J., Sullivan, J., Prevost, S., Churchill, E. (2000). Embodied Conversational Agents. MIT Press, Cambridge, MA, USA.

Cassell, J. (2000a). Embodied Conversational Agents, In: Communication of ACM. Vol. 43, No. 4. Page 70-78. ACM, New York.

Ekman, P., Friesen, W.V., Ellsworth P. (1972). Emotion in the Human Face. Cambridge University Press, Cambridge, USA.

Hachet, M., Guitton, P. (2002). The interaction table: a new input device designed for interaction in immersive large display environments. In: Proceedings of the 16th annual ACM symposium on User interface software and technology. Page 189-196. ACM Press, New York.

Heylen, D. et al. (2002). Experimenting with the Gaze of a Conversational Agent. In: Proceedings of the International CLASS Workshop on Natural, Intelligent and Effective Interaction in Multimodal Dialogue System. Page 93-100. Copenhagen.

Johnson, W.L., Rickel, J.W., Lester, J.C. (2000). Animated Pedagogical Agents: Face-to-Face Interaction in Interactive Learning Environments. In: International Journal of Artificial Intelligence in Education. No. 11, Page 47-78.

Krämer, N., Bente, G. (2003). Brauchen Interface Agenten Emotionen? In: Mensch und Computer 2003, Interaktion in Bewegung. Ziegler, J., Szwillus G. (Ed.), Page 287-296. Teubner, Stuttgart.

Krzysztof, G., Kulkarni, A. (2001). FIRE: An Information Retrieval Interface for Intelligent Environments. In: Proceedings of International Workshop on Information Presentation and Natural Multimodal Dialogue IPNMD'01. Verona, Italy. 
Pease, A., (2003). Light and Displays Highlights. In: Picture of the Future. Page 32-34. Siemens, Munich.

Picard, R.W. (1998). Affective Computing. MIT Press, Cambridge, MA, USA.

Wahlster, W., Reithinger, N., Blocher, A. (2001). SmartKom: Towards Multimodal Dialogues with Anthropomorphic Interface Agents. In: MTI Statustagung. DFKI, Saarbrücken, Germany. 